\title{
Retrospective Revision of Knee Arthroplasty Performed using Robotically Assisted Technique
}

\author{
Perazzini $\mathrm{PG}^{1}$, Conti $\mathrm{G}^{2 *}$, Sembenini $\mathrm{P}^{1}$, Marangon $\mathrm{A}^{1}$ and Alberton $\mathrm{F}^{1}$ \\ ${ }^{1}$ San Francesco Clinic SpA, Italy \\ ${ }^{2}$ Department of Neurosciences, University of Verona, Italy \\ *Corresponding author: Giamaica Conti, Department of Neurosciences, Biomedicine and Movement, University of Verona, Italy
}

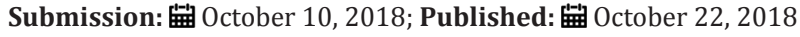

\begin{abstract}
Minimally invasive unicompartimental knee arthroplast is characterized by numerous advantages, in comparison with total knee arthroplasty, such as earlier recovery, less post-operative morbidity, improved knee function. These advantages have been obtained also thanks to the robotic assisted surgery, that allow to plan the arthroplasty more accurately, with improved outcome for patient. A device for robotically assisted arthroplasty that has emerging as the best device for unicompartimental knee arthroplasty. It works on compute tomography acquisitions and predicts the correct position for prosthesis, the ligament stress and the final extension and flection of the treated leg. In this study, we describe the results obtained on 876 patients subjected to different procedures in which the robotic device for knee arthroplasty has been employed to perform 919 knee implants. Statistic evaluation of different parameters, measured before and immediately after prosthesis placement, demonstrate the accuracy of robotic device for knee arthroplasty, in facilitate and assist surgeon procedures in order to obtain the best results for patients. By robotically assisted arthroplasty a negative outcome can be prevented because the positioning of the prosthesis is more correct and programed already before surgery, aligning rightly tibia and femur, balancing correctly the ligament and optimizing bone preparation.
\end{abstract}

Keywords: MAKOplasty; Unilateral knee arthroscopy; Robotically assisted surgery

\section{Introduction}

Unicompartmental knee arthroplasty (UKA) is a procedure to replace only one compartment of the diseased knee, most commonly the medial compartment [1]. The initial results of UKA were very encouraging but, later, proved disappointing and many surgeons abandoned the procedure. The causes of the early failures are multi-factorial and include poor patient selection and surgical technique, inadequate implant design, polyethylene wear, inaccurate instrumentation, poor understanding of the knee kinematics [2,3]. Despite this, during the last decades, UKA had known a continuous development due to the progression of the technologies by which the arthroplasty was performed and the improvement also of material of prosthesis [4,5]. UKA is indicated for non-inflammatory, noninfectious knee arthritis that affects one compartment. The knee must have minimal contractures and correctable mal-alignment [5]. The patient's body weight and bone quality must be evaluated before surgery, and the restrictions and expectations need to be discussed with patients. The introduction of minimally invasive UKA by John Repicci (Buffalo, NY), had proved beneficial in minimizing soft tissue trauma with UKAs. Patients had shown earlier recovery, improved knee function and greater range of motion, with a strong reduction of post-operative morbidity [68].
The advantages of UKA include smaller incision, reduced muscle dissection, leading to lesser postoperative pain, quicker rehabilitation, reduced blood loss, lower infection rate, preservation of range of motion and lower cost. Retention of both cruciate ligaments may also provide better knee kinematics as compared to total knee arthroplasty [9]. Despite the large number of studies regarding the minimally invasive UKA $[10,11]$, only few of them described a new interesting robotic technique named roboticassisted arthroplasty [12-14]. In this work it has been reported a retrospective study aimed to evaluate on the device used in robotic assisted UKA and the technique based on its employment. Only recently, this technique was described and employed for UKA $[15,16]$. In this retrospective study all the post-operative progresses of 919 patients, subjected to UKA in the period between January 2011 and May 2016, were described in order to analyze the improvements provide by robotically assisted arthroplasty.

\section{Materials and Methods}

\section{Patients}

Eight hundred seventy six patients (351 men and 525 women) were subjected to robotically assisted surgery for knee osteoarthritis. Nine hundred nineteen knee implants were placed 
and, precisely they could be divided in 768 medial, 85 lateral, 56 bicompartmental, 6 patellar femoral and 4 LCA reconstruction medial makoplasty. Forty three patients were underwent to bilateral one step implant. All the patients were subjected to spinal anesthesia (only few cases general).

During surgical procedures robotic device registered the following parameters, in order to compare, directly, the obtained results with the initial situation of the patient knee:
A. Initial extension
B. Initial flection
C. Final extension
D. Final extension
E. Variation in initial extension
F. Variation in initial flection
G. Variation in final extension
H. Variation in final flection

\section{Imaging of damaged areas}

All patients were subjected to magnetic resonance imaging (MRI) to evaluate the degree of damage and also a 3Dcomputed tomography (TC) is made before surgery using a specific protocol. The data collected with MRI e TC were employed to optimize the operative planning, but the robotically assisted arthroscopy allowed to select the position of prosthesis intraoperatively and the robotic arm gives real-time tactile feedback after the surgery.

\section{Minimally invasive robotically assisted surgical tech-} nique

The robotic device for knee arthroplasty (Figure 1) consists in a robotic arm, controlled by the software, that determine bone, femoral and tibia contours. The treated leg must be stabilized during all the procedure (using a leg holder) with the possibility to move the articulation in order to guarantee the minimally invasive access. Successively, it was necessary to identify with a probe some anatomical reference points in order to map bone surface and obtain a perfect overlap of bone geometry with the virtual knee reconstruction (Figure 2). Trough a small incision, (from 6 to $10 \mathrm{~cm}$ ) was possible to remove all the osteophytes (femoral, tibia and patellar) that create a ligament tension, or represent an impediment during the movement of knee in all directions (the first important step of the pre-operative procedure). At this point, the surgeon moved the knee applying a constant ligament stress in varus/valgus and the robotic device registered a graph (the second and most difficult step of the pre-operative procedure) during the full rotation of the knee. The graph showed the distance between the implant components and the soft tissues.

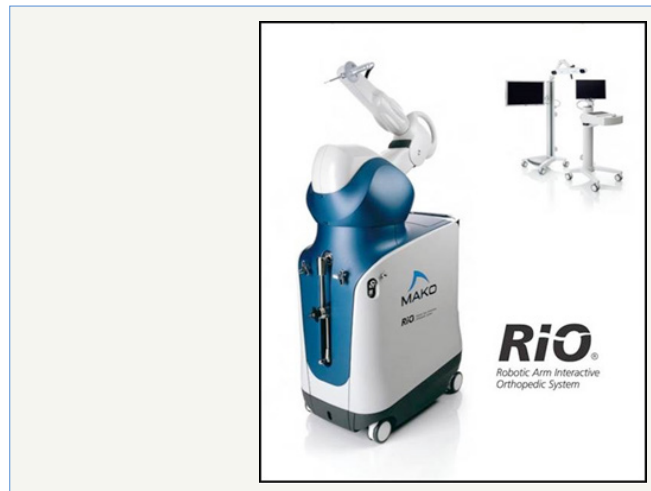

Figure 1: Mako ${ }^{\circledR}$ Rio Robot. The figure shows the structure of MAKO ${ }$ apparatus.

The possibility to adjust the implant placement site, moving all the components that compose the articulation, allowed to obtain a correct flexion-extension ratio and the balancing of ligaments strength, in order and to predict the final result (Figure 2). A cutting burr, applied at the finish of the robotic arm, controlled by the software and handled by the surgeon, allowed to resect the volume of bone, exactly corresponding to the shape of the prosthesis. After the placement of prosthesis the robotic device provided also the alignment of limbs, about the overlapping of the implants and information about knee rotation movements. All the parameters had showed on the computer screen and surgeon had a real time feedback on the correct positon of the prosthesis. The cementation is the final step of the surgery (Figure 3). One month after surgery the placement of prosthesis was checked by radiographic imaging Figure 4.

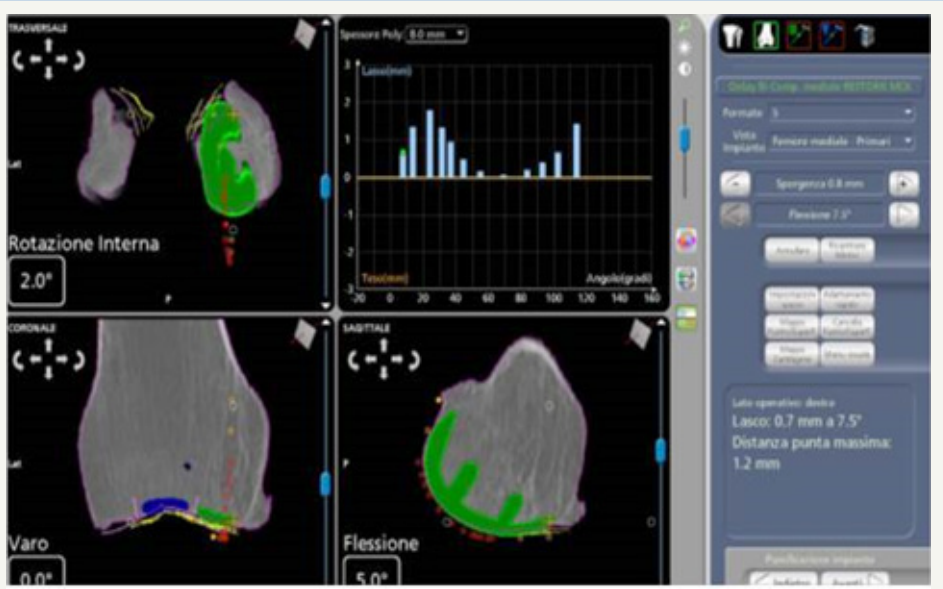

Figure 2: The 3D reconstruction of prosthesis positioning following MAKO ${ }^{\circ}$ predictions. 


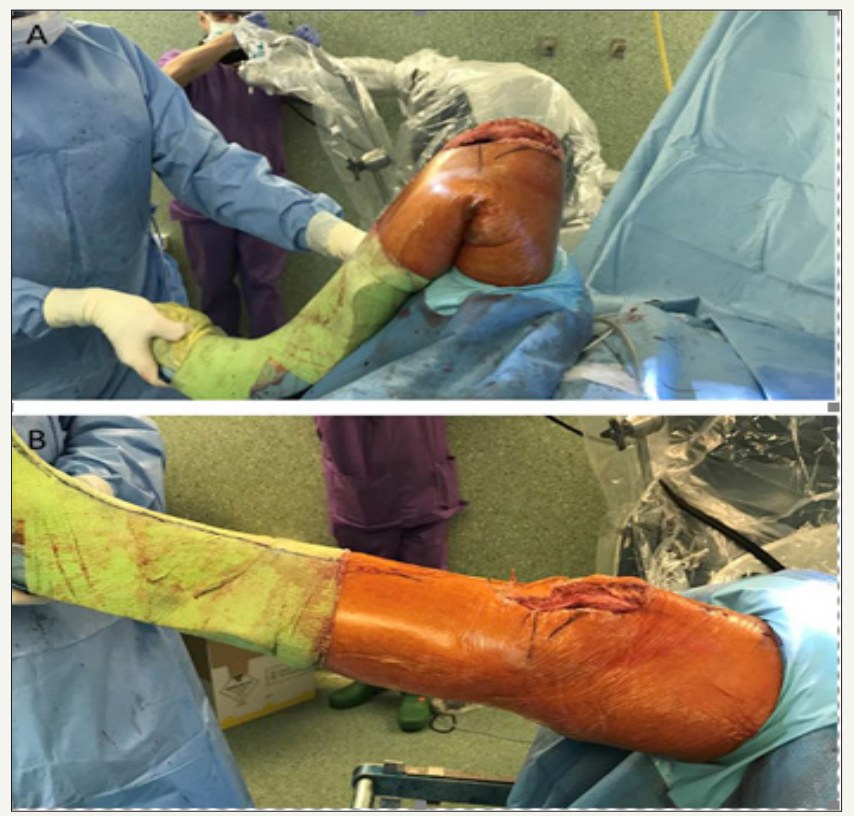

Figure 3: Full flexion and extension knee ROM. The figure shows the results of flection (A) and extension (B) of leg immediately after surgery.

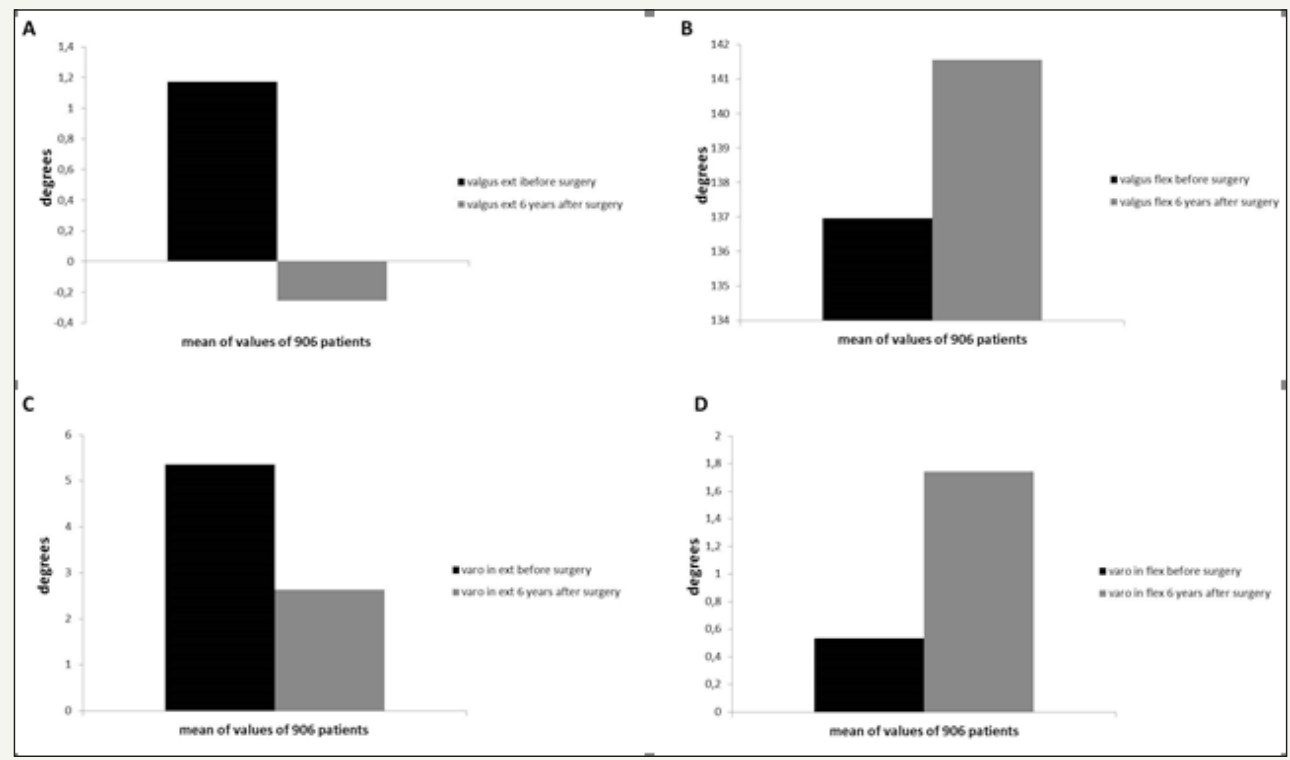

Figure 4: Data collected about flexion and extension degrees improvement six years after the surgery.

\section{Result}

Eight hundred seventy six patients, subjected to robotically assisted knee arthroplasty, were examined immediately after surgery and one, two, three, four, five and six years later. The parameter controlled and registered were varo/valgus extension and flexion, compared with the value registered by robotic device before surgery. The Figure 4 shows the graphs of the improvement of knee function in extension and flexion after surgery. The differences in the values before and after six years of follow up are statistically significant with a p value of 0.00001 . Exactly the mean value of flexion improvement was of 4.5 degrees, while in extension of 1.4 degrees with a correction of varo of 2.72 degrees.

\section{Discussion}

The introduction of robotically assisted surgery for UKA, was not easily accepted by surgeons, especially by those who employed standard surgical procedure for more time. They perceived a robot as a kind of opponent, that would replace the surgeons. The more experienced surgeons were not immediately convinced about the potential of robotic surgery, as conscious of their capability in the knee arthroplasty. With the introduction of the robotically assisted surgical technique it is possible to collect more accurate data that allow to the surgeon to plan correctly the placement of prosthesis and predict the result. Moreover, at the end of surgery, the robot allowed to verify the placement of prosthesis and evaluates the parameters of extension and flexion immediately after surgery, 
providing detailed information on patients outcome. Actually the best device could be considered the MAKO ${ }^{\circledR}$ that allows, to the surgeon, to have a detailed simultaneous description of the injured area and of the final result.

The robot provides numeric data, grades of the movement angles and the voltage graphs, but there are other aspects which are managed directly by the surgeon, such as the positioning of the prosthesis and the ligament tension. Traditional surgery is unable to evaluate the outcomes before surgery and it is not capable to calculate some factors such as the size and the bulk in the periphery of the prosthesis. All these factors lead to severe disorders. On the other hand, data provided by $\mathrm{MAKO} \AA$, require considerable experience for the correct interpretation. MAKO $®$ is characterized by the combination of pre-surgical planning made using an axial computed tomography (CT) scanning, combined with robotic arm. The combination with CT allowed to perform tridimensional reconstruction of injured area in which the surgeon must positioned the prosthesis. By robotically assisted surgery a negative outcome can be prevented because the positioning of the prosthesis is calculated before surgery, aligning rightly tibia and femur, balancing correctly the ligament and optimizing bone preparation. In the present study a large number of patients and different interventions were described, in order to verify the potentiality of robotic device in knee arthroplasty improvement.

Basing on the results obtained from patients treated with robotically assisted surgery it is possible to confirm the high prediction capability of device. In fact, for all the 906 patients there was an high statistical significance in term of extension and flection improving. Obviously the improvement in extension and flection after knee arthroplasty depends also on other factors dependent by the surgeon, that robotic device cannot calculate. Despite this aspect, the importance of robot in knee arthroplasty is unconfutable due to the capability to prevent bad prognosis and to promote a correct positioning of prosthesis and to calculate important parameters that help the surgeons in the clinical practice.

\section{Conclusion}

This study demonstrate the importance of $\mathrm{MAKO} \AA$ in UKA due to the possibility of chosing the most specific type of prosthesis, and allows to place the prosthesis more accurately and with a better prognosis, in comparison with the traditional arthroscopy. The experience of surgeons remains indisputable and, surely, contributes to the positive outcome. With MAKO® it is possible to realize the best combination between technology and surgeons experience in the field of knee arthroplasty.

\section{Figures and Captions to Figures}

The figure shows the final 3D reconstruction provided by $\mathrm{MAKO} \otimes$, that predict the most correct prosthesis placement following the extrapolation of data by software and after the application of ligaments tension. The graphs showed the improvement of flexion and extension capability determined 6years after the knee arthroplasty, providing the amount of degrees measured by robotic device during knee movement.

\section{References}

1. Kasodekar VB, Yeo SJ, Othman S (2006) Clinical outcome of unicompartmental knee arthroplasty and influence of alignment on prosthesis survival rate. Singapore Med J 47(9): 796-802.

2. Laskin RS (1978) Unicompartmental tibiofemoral resurfacing arthroplasty. J Bone Joint Surg Am 60(2): 182-185.

3. Bernasek TL, Rand JA, Bryan RS (1988) Unicompartmental porous coated anatomic total knee arthroplasty. Clin Orthop Relat Res 236: 5259.

4. Roche M (2014) Robotic-assisted unicompartmental knee arthroplasty: the MAKO experience. Clin Sports Med 33(1): 123-132.

5. Roche M (2015) Robotic-assisted unicompartmental knee arthroplasty: the MAKO experience. Orthop Clin North Am. 46(1): 125-131.

6. Repicci JA, Hartman JF (2004) Minimally invasive unicondylar knee arthroplasty for the treatment of unicompartmental osteoarthritis: an outpatient arthritic bypass procedure. Orthop Clin North Am 35(2): 201-216.

7. Repicci JA, Eberle RW (1999) Minimally invasive surgical technique for unicondylar knee arthroplasty. J South Orthop Assoc 8(1): 20-27.

8. Romanowski MR, Repicci JA (2002) Minimally invasive unicondylar arthroplasty: eight-year follow-up. J Knee Surg 15(1): 17-22.

9. Yang KY, Wang MC, Yeo SJ, Lo NN (2003) Minimally invasive unicondylar versus total condylar knee arthroplasty--early results of a matched-pair comparison. Singapore Med J 44(11): 559-562.

10. Lombardi AV, Berend KR, Berend ME, Della Valle CJ, Engh GA, et al. (2012) Current controversies in partial knee arthroplasty. Instr Course Lect 61: 347-381.

11. Repicci JA (2003) Mini-invasive knee unicompartmental arthroplasty: bone-sparing technique. Surg Technol Int 11: 282-286.

12. Lonner JH (2009) Indications for unicompartmental knee arthroplasty and rationale for robotic arm-assisted technology. Am J Orthop (Belle Mead NJ) 38(2): 3-6.

13. Sinha RK (2009) Outcomes of robotic arm-assisted unicompartmental knee arthroplasty. Am J Orthop (Belle Mead NJ) 38(2 Suppl): 20-22.

14. Roche M, O'Loughlin PF, Kendoff D, Musahl V, Pearle AD (2009) Robotic arm-assisted unicompartmental knee arthroplasty: preoperative planning and surgical technique. Am J Orthop (Belle Mead NJ) 38(2 Suppl): 10-15.

15. Werner SD, Stonestreet M, Jacofsky DJ (2014) Makoplasty and the accuracy and efficacy of robotic-assisted arthroplasty. Surg Technol Int 24: 302-306.

16. Pearle AD, Kendoff D, Stueber V, Musahl V, Repicci JA (2009) Perioperative management of unicompartmental knee arthroplasty using the MAKO robotic arm system (MAKOplasty). Am J Orthop (Belle Mead NJ) 38(2 Suppl): 16-19. 
Creative Commons Attribution 4.0 International License Journal

\section{Benefits of Publishing with us}

- High-level peer review and editorial services

- Freely accessible online immediately upon publication

- Authors retain the copyright to their work

- Licensing it under a Creative Commons license

- Visibility through different online platforms 